\title{
STELLAR POPULATIONS
}

Edited by P. C. VAN DER KRUIT and G. GILMORE
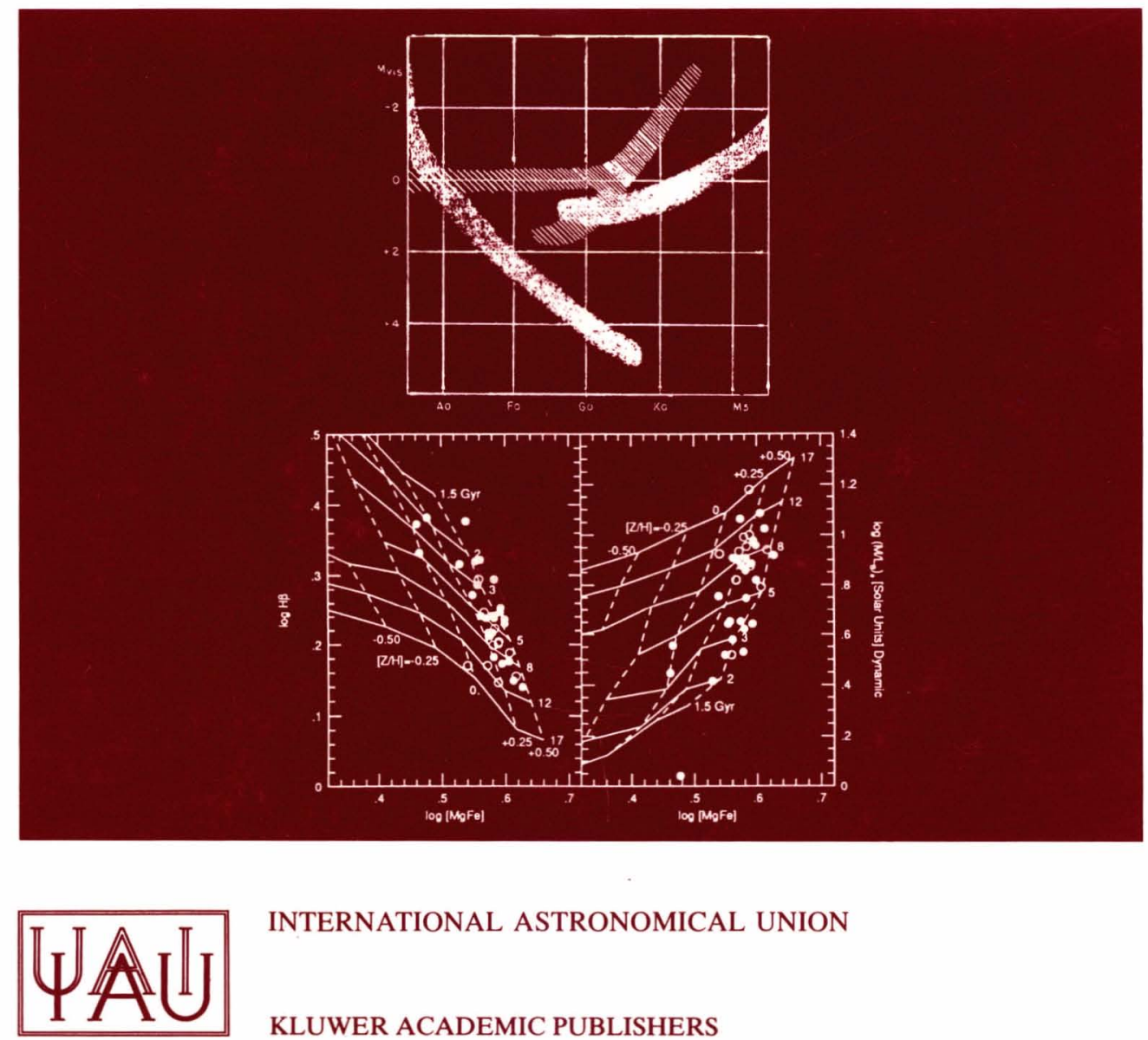

INTERNATIONAL ASTRONOMICAL UNION

KLUWER ACADEMIC PUBLISHERS 
STELLAR POPULATIONS 


\title{
STELLAR POPULATIONS
}

PROCEEDINGS OF THE 164TH SYMPOSIUM OF THE INTERNATIONAL ASTRONOMICAL UNION, HELD IN THE HAGUE, THE NETHERLANDS, AUGUST 15-19, 1994

\author{
EDITED BY \\ P.C. VAN DER KRUIT \\ Kapteyn Astronomical Institute, \\ University of Groningen, The Netherlands \\ and \\ G. GILMORE \\ Institute of Astronomy, \\ University of Cambridge, U.K.
}

KLUWER ACADEMIC PUBLISHERS

DORDRECHT / BOSTON / LONDON

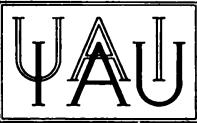


A C.I.P. Catalogue record for this book is available from the Library of Congress

\section{Published on behalf of \\ the International Astronomical Union \\ by}

Kluwer Academic Publishers, P.O. Box 17, 3300 AA Dordrecht, The Netherlands.

Kluwer Academic Publishers incorporates

the publishing programmes of

D. Reidel, Martinus Nijhoff, Dr W. Junk and MTP Press.

Sold and distributed in the U.S.A. and Canada

by Kluwer Academic Publishers,

101 Philip Drive, Norwell, MA 02061, U.S.A.

In all other countries, sold and distributed

by Kluwer Academic Publishers Group,

P.O. Box 322, 3300 AH Dordrecht, The Netherlands.

Printed on acid-free paper

\section{All Rights Reserved \\ C1995 International Astronomical Union}

No part of the material protected by this copyright notice may be reproduced or utilized in any form or by any means, electronic or mechanical including photocopying, recording or by any information storage and retrieval system, without written permission from the publisher.

Printed in the Netherlands 


\section{CONTENTS}

Preface

xiii

History of the Concept of Stellar Populations

OWEN GINGERICH: Report on the Progress in Stellar Evolution to $1950 \ldots \ldots \ldots \ldots \ldots \ldots \ldots$

DONALD E. OSTERBROCK: Walter Baade's Discovery of the Two Stellar Populations . . . . . . . . . . . . . .

NANCY GRACE ROMAN: The Discovery of the Chemical Composition - Kinematics Connection in the 1950's . . . . . . A. BLAAUW: Stellar Evolution and the Population Concept after 1950; The Vatican Conference . . . . . . . . . . .

\section{Globular Cluster Systems}

JAMES E. HESSER: The Galactic Globular Cluster System . .

F. FUSI PECCI and G. CLEMENTINI: The Stellar Population of a Typical Globular Cluster . . . . . . . . . . . . . . 67

T.D. KINMAN: Stars in the Galactic Halo . . . . . . . . . 75

WILLIAM E. HARRIS: Globular Cluster Systems in Other Galaxie

Open Clusters and the Galactic Disk

GERARD GILMORE: Evolution of the Galactic Halo and Disk

P.E. NISSEN: Age and Metallicity Distributions among Galactic

Disk Stars . . . . . . . . . . . . . . . . 109

K.C. FREEMAN: Early-Type Stars . . . . . . . . . . . 119

FELIX J. LOCKMAN: High Velocity Clouds in the Galaxy . . . 129

ROSEMARY F.G. WYSE: Chemical Evolution of the Galactic

Disk and Bulge ................ 133

Stellar Populations in Local Group Galaxies 151

M.W. FEAST: Stellar Populations in the Magellanic Clouds . . 153

W.L. FREEDMAN: M31 and Companions: A Key to Baade's

Stellar Populations Then and Now . . . . . . . . . . . 165

ABHIJIT SAHA: The Stellar Populations in Local Group Dwarf Galaxies . . . . . . . . . . . . . . . . . . 175

EDWARD W. OLSZEWSKI: Star Clusters in Local Group Galaxies 
Dark Matter in Galaxies 193

KONRAD KUIJKEN: Dark Matter in the Milky Way . . . . . . 195

P.C. VAN DER KRUIT: Dark Matter in Spiral Galaxies . . . . 205

P.T. DE ZEEUW: Central Black Holes and Dark Halos in Elliptical Galaxies . . . . . . . . . . . . . . . . 215

CLAUDE R. CANIZARES: Dark Matter in Clusters of Galaxies 227

Stellar Populations in Elliptical Galaxies $\quad \mathbf{2 3 7}$

H.G. FERGUSON: The UV Upturn in Elliptical Galaxies . . . . 239

S.M. FABER, S.C. TRAGER, J.J. GONZALEZ and GUY WORTHEY: The Stellar Ages of Elliptical Galaxies . . . . . . . . 249

R. BENDER and A. PAQUET: Stellar Populations in S0 Galaxies . . . . . . . . . . . . . . . 259

MARIJN FRANX: Measuring the Evolution of the M/L Ratio from the Fundamental Plane . . . . . . . . . . . . . 269

FRANCOIS SCHWEIZER: Effects of Late Mergers . . . . . . . 275

J.R. LUCEY: Environmental Effects in the Stellar Populations of Elliptical Galaxies . . . . . . . . . . . . . . . . 281

Galaxies at Large Redshifts

289

RICHARD S. ELLIS: The Morphological Evolution for Field Galaxies . . . . . . . . . . . . . . .

ROBERT W. O'CONNELL: Stellar Populations at Large Red-

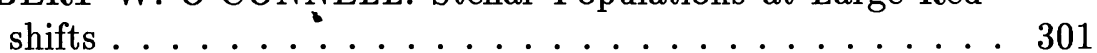

J.P. GARDNER: Galaxy Evolution from Deep Galaxy Counts . 311

Classification Schemes of Stellar Populations 321

S.D.M. WHITE: Formation Models of Galaxies . . . . . . . . . 323

ALVIO RENZINI: Stellar Dating and Formation of Galactic Spher-

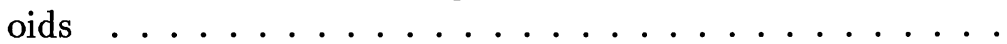

IVAN R. KING: Panel Discussion on the Classification and Terminology of Stellar Populations . . . . . . . . . . . . 337

JEREMY MOULD: Conference Summary . . . . . . . . . . . 349

Poster Papers

Saul J. Adelman and A. G. Davis Philip: Elemental Abundances of Population I Main Sequence B, A and F Stars and Population II A Stars . . . . . . . . . . . . . . . .

K.P. Tsvetkova, M.K. Tsvetkov and K.Y. Stavrev: Flare Stars Database II . . . . . . . . . . . . . . . . . 360

M. Chavez, M.L. Malagnini and C. Morossi: New $\mathrm{Mg}_{2}$ Indices from New Model Atmospheres. . . . . . . . . . . . . 
T. Shvelidze and V. Malyuto: Automated Spectral Classification of Stars by Means of Objective Prism Spectra . . . . . . . 362

D. Schaerer, W. Schmutz and A. de Koter: Complete Stellar Models: Interior and Spectral Evolution of Massive Stars . .

Jiang ShiYang and Liu YanYing: The Population and Evolution of A to F Pulsating Stars

363

364

Valeri Malyuto: New Algorithms to Calibrate the Strömgren Photometric System in Terms of Metal-to-Hydrogen Ratio . . . 365

A. Arellano Ferro, L. Parrao and L. Mantegazza: Supergiant Stars as Tracers of Galactic Chemical Composition . . . . . 366

C.A.L. Jones and T. von Hippel: Automating the Classification of Stellar Spectra . . . . . . . . . . . . . . . . 367

A. Alonso, S. Arribas and C. Martinez-Roger: Colours and Effective Temperatures of Main Sequence Stars Covering the Galactic Metallicity Range... . . . . . . . . . . .

J. Anosova, J. Colin and L. Kiseleva: On the role of Triple Systems in the Dynamics of the Galaxy: Stars with High Velocities

L. Kiseleva, J. Anosova, P.P. Eggleton, J. Colin and V. Orlov: Stability and Instability of Hierarchical Triple Stars . . . .

J. Anosova, K. Tanikawa and Y. Funato: Binaries in the Universe

J. Anosova and L. Kiseleva: Isolated Binaries and Triples in Nbody Systems . . . . . . . . . . . . . .

R.L. Smart: Kinematics of Orion Association Members . . . . E. M. Nezhinskij, L. P. Ossipkov and S.A. Kutuzov: Open Cluster System: Kinematics, Orbits . . . . . . . . . . . .

C. Jordi, E. Trullols, D. Galadi-Enríquez and F. Lahulla: Cepheus OB3: Search for Faint Members . . . . . . . . . . . .

M. Manteiga Outeira, J.R. Acarreta Rodriguez, C. Martinez Roger and O. Straniero: BVRI CCD Photometry of the Open Cluster IC166

G.N. Salukvadze and G. Sh. Javakhishvili: Some Physical Parameters and $u v b y \beta$ Photometry of Trapezium-Type Multiple Systems . . . . . . . . . . . . . .

E. Schilbach, H. Meusinger and J. Souchay: Luminosity and Mass Functions for the Pleiades from the Schmidt Survey . . . .

S.D. Wiramihardja, M. Nakano and T. Kogure: Surveys of Emission-Line Stars in the Outer Part of the Orion Star-Forming

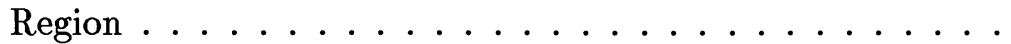

G.A. Medina Tanco and J.E. Steiner: Galactic Distribution of Symbiotic Stars . . . . . . . . . . . . . . . 
O. Yu. Malkov: Another Source of Local Missing Mass . . . . . 383

Devendra K. Ojha, Annie C. Robin and Olivier Bienaymé: The Asymmetric Drift of the Thick Disk Population . . . . . . . V.Straižys and A.G. Davis Philip: A Project for Investigation of the Stellar Population of the Galactic Disk . . . . . . . .

C. Soubiran, M.N. Perrin, R. Cayrel and E. Chereul: A New Sample of Thick Disk and Halo Stars . . . . . . . . . . .

I.F. Bikmaev: Metallicity Distribution and Kinematics of the Disk and Halo F-G Stars . . . . . . . . . . . . . . A.C. Robin, M. Haywood, D.K. Ojha, O. Bienayme and M.

Crézé: The Thick Disc Population of the Galaxy . . . . .
M. Haywood, A.C. Robin and O. Bienaymé: The Vertical Structure of the Galactic Disc . . . . . . . . . . . . . . 389

C. Flynn and B. Fuchs: Density of Matter in the Galactic Disk . 390

S. Bartaši ütè: The Transition from Disk to Halo as seen from Correlations between Kinematics and Metallicity . . . . . .

G. Tautvaišiene: Investigation of Suspected Red Horizontal Branch Stars in the Galactic Field . . . . . . . . . . . . . . . 392

K.S. de Boer, M. Geffert, M. Odenkirchen, J.H.K. Schmidt, A. Theissen, J. Colin, C. Ducourant and J.-F. LeCampion: Are there Two Populations of sdB Stars Visible in Kinematic Samples?

Slobodan Ninković: An Estimate of the Dark Matter Content in

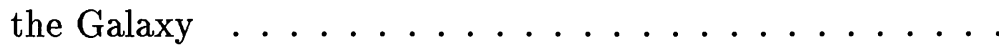

J. Borissova and N. Spassova: Pal 1: A Globular Cluster Anyway? . . . . . . . . . . . . . . . . .

B.F.W. Croke, R. Stathakis, R.D. Cannon, J.E. Hesser and R.A. Bell: Evidence for Primordial Abundance Variations in the Globular Cluster 47 Tuc . . . . . . . . . . . . . . .

K.J. Mitchell, R. Saffer and S.B. Howell: A New Sample of Faint Halo B Stars

Laura Kellar Fullton and Bruce W. Carney: The Age of the Galactic Inner Halo . . . . . . . . . . . . . . .

I. Platais, V. Kozhurina-Platais, M. Geffert and V.N. Frolov: Peripheral Members of M67 . . . . . . . . . . . . . . 399

B. Barbuy, S. Ortolani, A. Renzini, E. Bica and S. Castro: $\alpha$-Elements in Bulge Stars: Arp 1145 and Terzan 1-2 . . 400

J. W. Menzies: Three New Contact Binaries Near the Centre of NGC6121 (M4) . . . . . . . . . . . . . . . . . 401

Gang Zhao: Abundances of Heavy Elements in Halo Stars . . . . 402 K.S. de Boer, A. Theissen U. Heber and S. Möhler: The Scale Height of Blue Halo sdB Stars . . . . . . . . . . . . 403 
Andrew Layden and Robert Zinn: Kinematics of RR Lyrae Stars in the Inner (Old?) Halo . . . . . . . . . . . . . . . . . .

R.-D. Scholz, S. Hirte, M.J. Irwin and M. Odenkirchen: Kinematics of Galactic Globular Clusters from Schmidt-Plate Astrometry . . . . . . . . . . . . . . .

M. Geffert, B. Dauphole, J. Colin, M. Odenkirchen, H.-J. Tucholke and C. Ducourant: The Status of Absolute Proper Motions and the Kinematics of Globular Clusters . . . . . .

M. Catelan, B. Barbuy, J. A. de Freitas Pacheco, S. Ortolani and E. Bica: Evolutionary Aspects of the CMD in NGC 6553

404

G. de Marchi, F. Paresce and M. Romaniello: Low Mass Stars and White Dwarfs in NGC 6397 . . . . . . . . . . .

407

408

S. R. Zaggia, G. Piotto and M. Capaccioli: Mapping the Stellar Distribution in Globular Clusters: An Application to NGC $6809=\mathrm{M} 55 \ldots \ldots \ldots \ldots \ldots \ldots$. . . . . . . .

A. Ruelas-Mayorga and G. Garcia-Ruiz: Strömgren Photometry of Stars in Baade's Window . . . . . . . . . . . . . . 410

W.K. Griffiths, I.N. Kanatas, R.J. Dickens and A.J. Penny: CCD Photometry in the Globular Cluster M4 . . . . . . . P.W.Morris, W.K. Griffiths, I.N. Reid, A.J. Penny and R.J. Dickens: The Stellar Populations in the Disk of M31 . . . . Myung Gyoon Lee: Stellar Populations in the Pegasus Dwarf Gal-

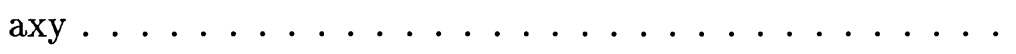

V. Friese, B. Fuchs and R. Wielen: Disks and Dark Haloes of Dwarf Spirals . . . . . . . . . . . . . . . . .

Ju. Frantsman and I. Shmeld: Two Different Stellar Populations in the Large Magellanic Cloud? . . . . . . . . . . . . . . . .

$V$. Hill: Chemical Content of Nine F-Supergiants from Different Regions of the LMC

Eline Tolstoy: The Resolved Stellar Populations of Leo A and GR8 . . . . . . . . . . . . . . . . . . . 417

C. Pryor, E.W. Olszewski and T.E. Armandroff: The Velocity Dispersions of the Draco and Ursa Minor Dwarf Spheroidal Galaxies . . . . . . . . . . . . . . . . . . .

A. Florsch, J. Marcout and J. Florsch: Size and Kinematic Aspects of the SMC According to the Catalogue of Bischoff Florsch - Marcout . . . . . . . . . . . . . .

J.P. Anosova and B.G. Anandarao: Dark Matter Inside and Around Binary Galaxies: Formation of Interacting Galaxies

A. Just, B. Fuchs and R. Wielen: The Vertical Age Distribution of Stellar Disk Populations in Edge-on Galaxies . . . . . . . 423 
Uta Fritze-v. Alvensleben: Star and Globular Cluster Formation in Mergers . . . . . . . . . . . . . . . . . . . . . 424

Annette Ferguson, Rosemary Wyse, Jay Gallagher and Deidre Hunter: Where Does Star Formation End in NGC 1058 . . Claudia Möller, Klaus J. Fricke and Uta Fritz -v. Alvensleben: Metallicity of Stars and Gas in Galaxies of Different Hubble Types . . . . . . . . . . . . . . . . 426

Richard de Grijs and Piet C. van der Kruit: Edge-on Galaxies Dust Influence and Thick Disks . . . . . . . . . . . .

Gustavo Bruzual A. and Gladis Magris C.: Emission Lines in Star Forming Galaxies . . . . . . . . . . . . . . .

G. Mackie, G. Fabbiano, D.-W. Kim and Y. Ikebe: Local and Global Optical, Far-Infrared (FIR) and X-ray Properties of the FIR quiescent Sc Galaxy NGC 247 . . . . . . . . . . Elchanan Almoznino and Noah Brosch: Stellar Populations of Dwarf Galaxies . . . . . . . . . . . . . . . . J.R. Mould, W. Freedman, L. Ferrarese and D. Kelson: Discovery of Cepheids in M100 and M101 . . . . . . . . . . D.F. de Mello, W.C. Keel, J.W. Sulentic and R. Rampazzo: Stellar Populations in Mixed Pairs of Galaxies . . . . . . . . . . 434

Wim van Driel: Dark Matter in the Polar Ring Spiral NGC 660 M. Sodemann and B. Thomsen: Surface Brightness Fluctuations and the Stellar Population in Elliptical Galaxies ...... Olga K. Sil'chenko: New Metallicity Scale of Elliptical Galaxies Olga K. Sil'chenko: A Decoupled Nucleus in NGC 1052 . . . . . M. Serote-Roos, C. Boisson and M. Joly: A Study of the Stellar Populations ố NGC $3516 \ldots \ldots$. . . . . . . . . J. Loxen, U. Fritze - v. Alvensleben, K.J. Fricke and G. Hensler: Chemodynamic and Photometric Evolution of Elliptical Galaxies . . . . . . . . . . . . . . . . . . . 440

Judith G. Cohen: The M87 Globular Cluster System . . . . . . . 441

M.A. Pahre, S. Djorgovski, K. Matthews, D. Shupe, R. de Carvalho, and J.R. Mould: Near-IR Imaging of Elliptical Galax-

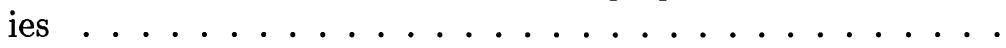

F. Bertola, C. Chiosi, A. Bressan, L.M. Buson, D. Burstein, S. di Serego Alighieri: HST Far-UV Imaging of M31, M32 and NGC 205

F. Bertola, M. Cappellari, D. Burstein, L. Greggio, A. Renzini and S. di Serego Alighieri: UV Central Spikes in Early Type Galaxies . . . . . . . . . . . . . . . . . . .

A. Pizzella, R. Morganti, E.M. Sadler and F. Bertola: The Dark Massive Halo in the Elliptical Galaxy NGC $5266 \ldots \ldots 446$ 
Pascale Jablonka: Metallicity of NGC 5128 Globular Clusters . .

Young-Wook Lee and Jang-Hyun Park: The Age Spread among Galaxies and the Origin of the UV Radiation from Elliptical Galaxies . . . . . . . . . . . . . . . . . . . . . . . 448

Noah Brosch: The UV Content of Virgo Cluster Galaxies . . . 450

T.K. Chatterjee: Enhanced Star Formation rates in Binary Interacting Elliptical Galaxies . . . . . . . . . . . . . 452

D. Fisher, G. Illingworth and M. Franx: Line Strength Profiles in Early-Type Galaxies . . . . . . . . . . . . . . . .

M. Kalinkov, I. Kuneva, and I. Valtchanov: Dark Matter in Groups and Clusters of Galaxies . . . . . . . . . . . . .

Konstantin P. Sokolov: Radio Source Counts at Ultra-Low Fre-

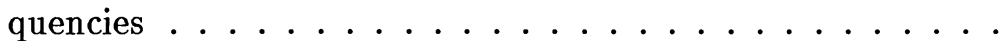

J.P. Anosova, P.M.S. Namboodiri and M.R. Deshpande: Formation of $\mathrm{cD}$-Galaxies and Gravitational Lenses: Computer Simulations ....................

Uta Fritze-v. Alvensleben and Klaus J. Fricke: Metal Abundances of Damped Ly $\alpha$ Systems and the Chemical Evolution of Spiral Galaxies .................... . .

A. Milone and B. Barbuy: Synthetic Spectra for Single-Aged Populations ...................... . .

Pascale Jablonka and D. Alloin: Blue Galaxies in Distant Clusters . . . . . . . . . . . . . . . . .

B.M. Poggianti and G. Barbaro: Determination of Young Population Ages in Cluster Galaxies . . . . . . . . . . . . .

C. Robert, C. Leitherer, and T.M. Heckman: Ultraviolet Population Synthesis of Starburst Galaxies Observed with HST . .

C. Rola, L. Tresse, G. Stasinska and F. Hammer: The Nature of the Emission Galaxies in the Canada France Redshift Survey - CFRS ....................

J.B. Hutchings, R. Abraham, T. Smecker-Hane, S. Morris, T. Davidge, M. Rigler, R. Carlberg, H. Yee and E. Ellingson: Populations in the $\mathrm{z}=0.23$ Rich Cluster Abell $2390 \ldots 463$ 